\title{
Hyperthermia Induced by Open-Field Stress Is Blocked by Salicylate ${ }^{1}$
}

\author{
REBECCA SINGER, CHRISTIAN T. HARKER, \\ ARTHUR J. VANDER AND MATTHEW J. KLUGER ${ }^{2}$ \\ Department of Physiology, The University of Michigan Medical School \\ Ann Arbor, MI 48109
}

Received 11 September 1985

\begin{abstract}
SINGER, R., C. T. HARKER, A. J. VANDER AND M. J. KLUGER. Hyperthermia induced by open-field stress is blocked by salicylate. PHYSIOL BEHAV 36(6) 1179-1182, 1986.-Psychological stress results in a rise in body temperature. Here we report that in rats, hyperthermia induced by open-field stress can be blocked by administration of the antipyretic drug sodium salicylate. These data suggest that this rise in body temperature is a true fever, perhaps mediated by prostaglandins.
\end{abstract}

Hyperthermia Sodium salicylate Interleukin-1 Prostaglandins Fever Temperature regulation

PSYCHOLOGICAL stress results in an acute rise in body temperature in rats $[1,4,12,13,22,26,28]$, rabbits $[21,27]$ and people $[15,20]$. Exposure to novel environments and handling can produce a rise in body temperature in rats of as much as $2^{\circ} \mathrm{C}[4,22]$. Although some studies have shown that part of this so-called "emotional hyperthermia" can be blocked by naloxone, an inhibitor of endorphins and enkephalins [1,22], Vidal [26] has concluded that endogenous opioids are not significantly involved in emotional hyperthermia. Hanukoglu [14] has suggested that prostaglandins may be the primary mediator of stress-induced hyperthermia, but to our knowledge, this hypothesis has not been tested.

Fever, the elevation in body temperature that occurs during infection, is thought to be mediated by the release of prostaglandins (primarily $\mathrm{PGE}_{1}$ and $\mathrm{PGE}_{2}$ ) in the central nervous system [9]. Drugs that attenuate fever, antipyretics, are thought to act by blocking the synthesis of these prostaglandins $[10,25]$. If prostaglandins are responsible for stressinduced hyperthermia then the fevers resulting from psychological stress should also be reduced by administration of an antipyretic drug.

We report that an injection of a dose of sodium salicylate that has no effect on the body temperature of nonstressed rats blocks the rise in body temperature of stressed rats. We conjecture that stress-induced hyperthermia may be initiated by the rapid release of prostaglandin in the area of the central nervous system responsible for regulating body temperature.

\section{METHOD}

\section{Animals}

Specific pathogen-free male Sprague-Dawley rats weigh- ing 200 to $300 \mathrm{~g}$ were obtained from Charles River Breeding Laboratories, Inc., Portage, MI. Rats were housed in individual plastic cages in a room maintained at $26 \pm 1^{\circ} \mathrm{C}$, i.e., in the thermoneutral zone for rats, with a photoperiod consisting of $12 \mathrm{hr}$ of light and $12 \mathrm{hr}$ of dark. Tap water and rodent chow (Purina) were provided ad lib.

\section{Measurement of Body Temperature}

Body temperature was measured using battery-operated biotelemetry devices (Mini-Mitter, Inc., Sunriver, OR) implanted intraperitoneally into each rat four or more days before the stress experiments began. Each transmitter was calibrated prior to implantation. Output (frequency in $\mathrm{Hz}$ ) was monitored by a mounted antenna placed under each animal's cage (or under the open-field box in Experiment 1; see below) and fed into a peripheral processor (Dataquest III Systems, Mini-Mitter, Inc.) connected to an IBM-PC. Temperatures were monitored and recorded at either 1 or 5 minute intervals. Prior to any experimental manipulation, basal temperatures were recorded for either 10 or 20 minutes.

\section{Stress Paradigm}

Acute psychological stress in rats can be induced by exposure to an open-field [7]. The open-field used in our experiments consisted of a $4 \times 4 \times 2 \mathrm{ft}$ high plywood box painted white on the inside and illuminated by a $200 \mathrm{~W}$ white light suspended from above [7]. The box was placed in a temperature-controlled environmental room near the environmental chamber housing the rats. The temperature within the open-field box was the same as the rat's home cages, $26^{\circ} \mathrm{C}$. The experimental protocol for the stress involved transporting each rat from the home room to the

'Research sponsored in part by ONR Contract N00014-K-0027.

${ }^{2}$ Requests for reprints should be addressed to Dr. Matthew J. Kluger. 


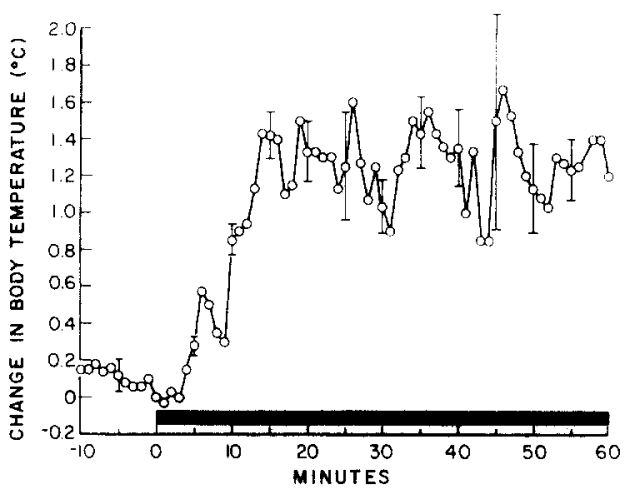

FIG. 1. Change in deep-body temperature $( \pm 1$ S.E. $)$ of five rats exposed to an open-field stress for 60 minutes beginning at time $=0$. Horizontal dark bar indicates stress period.

stress room, removing the rat from the cage and placing him into the open-field box. After the appropriate period of time the rat was returned to his cage and taken back to the housing area. Care was taken not to disturb the rats either before or after the stress-exposure. Control animals were not placed in the open-field box. All animals were conditioned to handling for at least 3 days prior to the experiments. To minimize possible circadian variability all exposure to the open-field occurred between 9 a.m. and 1 p.m.

\section{Experiment 1: Description of Stress-Induced Hyperthermia}

To characterize the pattern of rise in body temperature during exposure to the open-field, antennae were placed under the open-field box to allow for the continuous recording of deep body temperature. In this experiment, the temperatures of 5 rats were monitored at 1 minute intervals during a 60 minute exposure to the open-field.

Experiment 2: Effect of Injection of Saline, Naloxone or Sodium Salicylate on Stress-Induced Hyperthermia

Nineteen rats received intraperitoneal (IP) injections of 1 $\mathrm{ml}$ sterile pyrogen-free $0.9 \%$ sodium chloride (saline) 60 minutes prior to exposure to the open-field. Sixteen control rats also received injections of $1 \mathrm{ml}$ saline IP and were then returned to their home cages and kept there for the duration of the experiment. After the 30-minute exposure to the open-field, experimental rats were returned to their home cages and their temperatures monitored for the following hour. The saline injections were included in this protocol to permit comparison of these rats with those receiving drug injections.

In another series of experiments, two groups (a total of 32 rats) received IP injections of an antipyretic dose of sodium salicylate (Mallinckrodt, University Hospital Pharmacy, 200 $\mathrm{mg} / \mathrm{kg}$ ) dissolved in saline 60 minutes prior to exposure to the open-field. Fifteen of these rats were exposed to the openfield and 17 of the rats served as salicylate-treated unstressed controls.

To determine whether any portion of the hyperthermia might be attributable to an endogenous opioid as reported in several earlier studies $[1,22]$, rats were injected IP with naloxone (kindly provided by V. Nickolson, Dupont, 10 $\mathrm{mg} / \mathrm{kg}$ ) dissolved in saline. Twenty-six of these rats were exposed to the open-field and 20 rats served as naloxone treated unstressed controls.

Lastly, to determine whether sodium salicylate and naloxone might have additive or synergistic effects, rats were injected IP with the above doses of both these drugs 60 minutes prior to exposure to the open-field. Eighteen of these rats were exposed to the open-field and 16 rats served as sodium salicylate/naloxone-treated unstressed controk.

\section{Data Analysis}

Values reported are mean \pm 1 S.E. Comparisons between each experimental and control group were analyzed for statistical significance using the Student's t-test. All comparisons among groups were made using one-way ANOVA, followed by Scheffe allowances for multiple comparisons among means.

\section{RESULTS}

\section{Experiment /}

In Figure 1, the mean change in body temperature during open-field stress is plotted at 1 minute intervals for $10 \mathrm{~min}$ utes prior to the rats being put into the open-field and for the entire 60 minute period while in the open-field box. Within 14 minutes of exposure to the open-field, the temperature of these rats rose $1.43 \pm 0.27^{\circ} \mathrm{C}$. The body temperatures of the rats remained elevated for the 60 minute exposure to the open-field.

\section{Experiment 2}

The change in body temperature due to the stress period, calculated by subtracting the pre-stress temperature (temperature immediately prior to removing each rat from its cage) from the post-stress temperature (temperature immediately after returning the rat from the open-field to its cage) is shown in Fig. 2 for each group of rats and their control groups. Following the $\mathbf{3 0}$ minute stress period, the rise in body temperature of the rats injected with saline was $1.33 \pm 0.09^{\circ} \mathrm{C}$, significantly different from the change in body temperature of the control rats injected with saline and maintained in their home cages $(p<0.001$, Student's $t$-test). The fact that the rise in temperature observed in these stressed rats was no higher than that seen in rats of Experiment 1 indicates that saline injection did not alter the response or contribute to it.

Rats injected with naloxone and placed in the open-field had a rise in body temperature of $1.01 \pm 0.10^{\circ} \mathrm{C}$, significantly elevated from the change in body temperature of the control rats injected with naloxone and maintained in their home cages $(p<0.001$, Student's $t$-test). Although the rise in body temperature of rats injected with naloxone and exposed to the open-field tended to be less than in rats injected with saline and exposed to open-field, the decrease was not significantly different by use of ANOVA $(p>0.50)$.

Rats injected with sodium salicylate and exposed to the open-field had a rise in body temperature of $0.41 \pm 0.15^{\circ} \mathrm{C}$, significantly higher than the control rats injected with sodium salicylate $(p<0.002$, Student's $t$-test). The rise in body temperature of the rats injected with sodium salicylate and exposed to the open-field was significantly smaller than the rise in body temperature observed in the rats injected with either saline or naloxone and exposed to the open-field $(p<0.001$, ANOVA). 


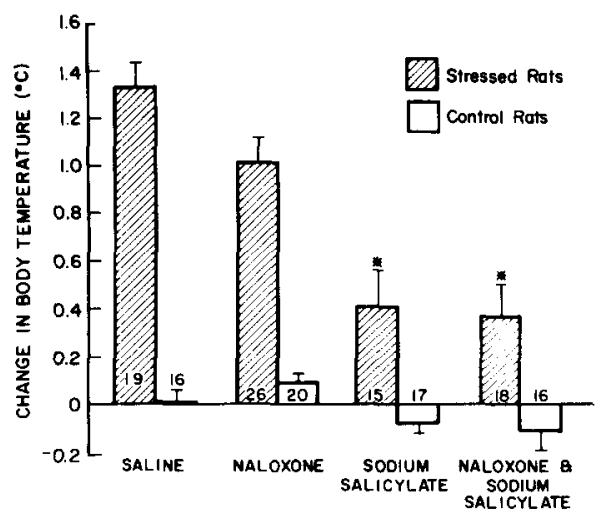

FIG. 2. Effect of saline, naloxone, sodium salicylate, and naloxone plus sodium salicylate on the change in deep-body temperature ( \pm 1 S.E.) in stressed and control rats. Stressed rats were exposed to an open-field for 30 minutes beginning at time $=0$. Control rats were left undisturbed in their cages for this period. The heights of the bars represent the differences in body temperature between time $=0$ and time $=30$ minutes. Numbers in or near each bar $=$ sample size. ${ }^{*}$ The stress-induced rise in body temperature in the sodium salicylateinjected group and in the sodium salicylate/naloxone injected groups were significantly different from that observed in either the saline- or naloxone-injected rats $(p<0.001)$.

Rats injected with both sodium salicylate and naloxone and exposed to the open-field had a rise in body temperature of $0.37 \pm 0.11^{\circ} \mathrm{C}$, similar to that observed in the rats injected with sodium salicylate alone and exposed to the open-field. This rise in temperature was significantly higher than that seen in control rats injected with salicylate and naloxone $(p<0.002$, Student's $t$-test). The rise in body temperature of the rats injected with these drugs and exposed to the openfield was smaller than the rise in body temperature observed in the rats injected with either saline or naloxone and exposed to the open-field ( $p<0.001$, ANOVA). Accordingly, this experiment and the one involving the use of naloxone alone, do not support the hypothesis that endorphins and enkephalins are the major contributor to the hyperthermia induced by open-field stress.

\section{DISCUSSION}

Since the administration of sodium salicylate blocked $69 \%$ of the peak stress-induced hyperthermia (body temperature rose $0.41^{\circ} \mathrm{C}$ vs. $1.33^{\circ} \mathrm{C}$ ) we hypothesize that a sizeable portion of the open-field stress-induced rise in body temperature is prostaglandin mediated. It is interesting that the rise in temperature occurs within minutes of exposure to the openfield (see Fig. 1). It is possible that exposure to stress results in the rapid release of prostaglandins within certain central nervous system sites. A recent study has shown that microinjections of PGE into the organum vasculosum of the laminae terminalis produce rises in body temperature as rapidly as those we report here [23].

A fever is defined as a rise in body temperature resulting from an elevation in the thermoregulatory set-point $[2,17]$. It is thought that the protein interleukin-1 (IL-1), responsible for modulating immune function and initiating the "acutephase response" to infection, is responsible for the development of fever $[3,8,16,18]$. IL-1 is believed to raise the thermoregulatory set-point in the hypothalamic region of the central nervous system by inducing the production of $\mathrm{PGE}_{\text {, }}$ and $\mathrm{PGE}_{2}[3,8,9,16,18]$. Since a large portion of stressinduced hyperthermia is blocked by the antipyretic drug sodium salicylate, we hypothesize that one acute response to psychological stress may be the release of IL-1. Interestingly, the onset of stress-induced hyperthermia is more rapid than that normally associated with fever induced by intravenous injection of IL-1. Therefore, if IL-1 does mediate stress-induced hyperthermia, it is likely to do so centrally [11], perhaps being produced locally in the hypothalamus, or in the organum vasculosum laminae terminalis [23].

IL-1 has recently been shown to be released during the stress of exercise $[5,6]$, and perhaps accounts for a small fraction of the rise in body temperature. Although the stimulus for the release of IL-1 in exercise is unknown, from a teleological viewpoint, the release of IL-1 in response to exercise makes sense. Since IL-1 plays a major role in facilitating both host defense and immune responses $[3,8$, $16,18]$, the release of this protein in response to the stress of exercise would prepare the organism for potential injury and infection, and therefore would be adaptive. This would be in keeping with the classical concept that many of the nonspecific responses to stress represent a "fight or flight" response, preparing the body for potential exercise and physical trauma. The same logic could be used for hypothesizing that psychological stress should result in the acute release of IL-1. To our knowledge, the present experiments are the first to evaluate this hypothesis, albeit indirectly.

\section{REFERENCES}

1. Blasig, J., V. Hollt, U. Bauerle and A. Herz. Involvement of endorphins in emotional hyperthermia of rats. Life $S_{c i}$ 23: 2525-2532, 1878.

2. Bligh, J. Temperature Regulation in Mammals and Other Vertebrates. Amsterdam: North-Holland Publ. Co., 1973.

3. Bornstein, D. L. Leukocyte pyrogen: a major mediator of the acute phase reaction. Ann NY Acad Sci 389: 323-336, 1982.

4. Briese, E. and M. G. De Quijada. Colonic temperature of rats during handling. Acta Physiol Lat Am 20: 97-102, 1970.

5. Cannon, J. G. and M. J. Kluger. Endogenous pyrogen activity in human plasma after exercise. Science 220: 617-619, 1983.

6. Cannon, J. G. and C. A. Dinarello. Increased plasma interleukin-1 activity in women after ovulation. Science 227: 1247-1249, 1985.
7. Clamage, D. M., C. S. Sanford, A. J. Vander and D. R. Mouw. Effects of psychosocial stimuli on plasma renin activity in rats. Am J Physiol 231: 1290-1294, 1976.

8. Dinarello, C. A. Interleukin-1. Rev Infect Dis 6: 51-95, 1984.

9. Feldberg, W. and A. S. Milton. Prostaglandins and body temperature. In: Handbook of Experimental Pharmacology, Volume 50// "Inflammation," edited by J. R. Vane and S. H. Ferreira. Berlin: Springer-Verlag, 1978, pp. 617-656.

10. Ferreira, S. H., S. Moncada and J. R. Vane. Indomethacin and aspirin abolish prostaglandin release from the spleen. Nature New Biol 231: 237-239, 1971.

11. Fontana, A., E. Weber and J.-M. Dayer. Synthesis of interleukin-1/endogenous pyrogen in the brain of endotoxintreated mice: a step in fever induction? $J$ Immunol 133: 16961698, 1984. 
12. Goldstein, A. and P. Lowery. Effect of the opiate antagonist naloxone on body temperature in rats. Life Si 17: 927-932, 1975.

13. Gollnick, P. D. and C. D. Ianuzzo. Colonic temperature re sponse of rats during exercise. J Appl Physio/ 24: 747-750, 1968.

14. Hanukoglu, I. Prostaglandins as first mediators of stress. N Engl J Med 296: 1414, 1977.

15. Hasan, M. K. and A. C. White. Psychogenic fever: entity or nonentity. Postgrad Med 66: 152-154, 1979.

16. Kampschmidt, R. F. Leukocyte endogenous mediator/endogenous pyrogen. In: Infection: The Physiologic and Metabolic Responses of the Host, edited by M. C. Powanda and P. G. Canonico. Amsterdam: Elsevier/North-Holland, 1981, pp. 55-74.

17. Kluger, M. J. Fever: Its Biology. Evolution and Function. New Jersey: Princeton University Press, 1979.

18. Kluger, M. J., J. J. Oppenheim and M. C. Powanda (editors) The Physiologic, Metabolic and Immunologic Actions of Interleukin-1. Proceedings of the International Symposium held in Ann Arbor, MI, June 4-6. 1985. In: "Progress in Leutocyle Biology." New York: Alan R. Liss. 1985, 586 pp.

19. Milton, A. S. and S. Wendlandt. Effects on body temperature of prostaglandins of the third ventricle of unanesthetized eats and rabbits. J Physiol (Lond) 218: 325-336, 1971.

20. Renbourn, E. T. Body temperature and pulse rate in boys and young men prior to sporting contests. A study in emotional hyperthermia: with a review of the literature. I Psychosom Res 4: $149-175,1960$
21. Snow. A. E. and A. Horita. Interaction of apomerphunc and stressors in the production of hyperthermia in the mbit. I Pharmacol Exp Ther 220: 335-339. 1982.

22. Stewart, J. and R. Eikelboom. Stress masks the hypothemic effect of naloxone in rats. Lifie $S_{i} i$ 25: $1165-1172,1979$.

23. Stitt, J. T.. S. G. Shimada and H. A. Bernheim. Microinjection of zymosan and lipopolysaccharide into the organum vasculosum laminae terminalis of rats enhances their febrile responsiveness to endogenous pyrogen. In: Thermal Phriviology. edited by J. R. S. Hales. New York: Raven Press. 1984. pp. $555-558$.

24. Ucar, D. A., R. J. Toeco and M. J. K'luger. Circadian variation in circulating pyrogen: possible role in resistance to intection. Proc Soc Exp Biol Mod 173: 319-323, 1983.

25. Vane. J. R. Inhibition of prostaglandin synthesis as a mechanism of action for aspirin-like drugs. Nuture $N$ in Birl 231: 232-235. 1971

26. Vidal, C. C. Suaudeau and J. Jacob. Hyper- and hypothermia induced by non-noxious stress: effects of naloxone, diazepam and gamma-acetylenic GABA. Life $S_{c i}$ 33: Suppl I. 587-590. 1983.

27. Yokoi, Y. Effect of ambient temperature upon emotional hyperthermia and hypothermia in rabbits. I Appl Physiol 21: 1795$1798,1966$.

28. York, J. L. and S. G. Regan. Conditioned and unconditioned influences on body temperature and ethanol hypothermia in laboratory rats. Pharmacol Biochem Behov 17: 119-124. 1982. 